\title{
Exploring the potential benefits of vaccinia virus complement control protein in controlling complement activation in pathogenesis of the central nervous system diseases ${ }^{\text {is }}$
}

\author{
Girish J. Kotwal ${ }^{\mathrm{a}, \mathrm{b}, *}$, Nilisha Fernando ${ }^{\mathrm{c}}$, Jianhua Zhou ${ }^{\mathrm{b}}$, Krisztina Valter ${ }^{\mathrm{c}, \mathrm{d}}$ \\ a Department of Medicine, University of Massachusetts School of Medicine, Worcester, MA, USA \\ ' Inflamed, Inc., Louisville, KY, USA \\ ${ }^{\mathrm{c}}$ The John Curtin School of Medical Research, Canberra, ACT, Australia \\ ${ }^{\mathrm{d}}$ Medical School, The Australian National University, Canberra, ACT, Australia
}

\section{A R T I C L E I N F O}

\section{Article history:}

Received 20 May 2014

Received in revised form 8 June 2014

Accepted 11 June 2014

Available online 19 July 2014

\begin{abstract}
A B S T R A C T
Aging is a major risk factor for the development of diseases related to the central nervous system (CNS), such as Alzheimer's disease (AD) and age-related macular degeneration (AMD). In both cases, linkage studies and genome-wide association studies found strong links with complement regulatory genes and disease risk. In AD, both CLU and CR1 genes were implicated in the late-onset form of the disease. In AMD, polymorphisms in $\mathrm{CFH}, \mathrm{CFB}$ and $\mathrm{C} 2$ were similarly implicated. The cost of caring for patients with AD or AMD is approaching billions of dollars, and with the baby boomers reaching their 60's, this amount is likely to increase further. Intervention using complement inhibitors for individuals in their early 50s who are at a higher risk of disease development, (testing positive for genetic risk factors), could slow the progression of $\mathrm{AD}$ or $\mathrm{AMD}$ and possibly prevent the severity of late stage symptoms. Although we have used the vaccinia virus complement control protein (VCP) to elucidate the role of complement in CNS diseases, it has merely been an investigational tool but not the only possible potential therapeutic agent.
\end{abstract}

(C) 2014 Elsevier Ltd. All rights reserved.

\section{Introduction}

The central nervous system has been described as the last frontier, and with regard to the understanding of the role of complement in both normal and diseased states, most of the progress made has only been in the past 2-3 decades. The pioneering work of the Tenner and Cooper groups reviewed 6 years ago (Alexander et al., 2008) on the possible role of complement in Alzheimer's disease (AD) was an impetus for others to investigate the precise role of complement in the pathogenesis of AD and to develop appropriate animal models and use complement inhibitors to evaluate the potential benefits of regulating complement. Complement system (CS) activation has been recognized to play an important role in the pathology and progression of many CNS conditions. In healthy human brain and retina, CS mRNA expression is normally low.

\footnotetext{
is This article belongs to SI: XXV ICW Rio 2014.

* Corresponding author at: University of Massachusetts School of Medicine, Department of Medicine, 4664 Shenandoah Drive, Louisville, KY 40241, USA. Tel.: +1 5023277466 .

E-mail address: gjkotw01@gmail.com (G.J. Kotwal).
}

However, in diseased brains and retina such as in Alzheimer's (AD), Huntington's and Pick's disease, Age-related macular degeneration (AMD), CS mRNA expression is markedly increased, especially in areas of primary pathology, while CS inhibitor levels show only minimal changes. In Huntington's, the pathological hallmark is apoptotic cell death of neurons in the putamen and caudate nuclei, in which both neurons and astrocytes show increased immunoreactivity for C1q, C4, C3 and C5b-9 (Singhrao et al., 1999; Zhou et al., 2008). This is further confirmed by a recent report that there is a dramatic increase in C1q protein levels in the brain (Stephan et al., 2013). Similarly, in AD, complement activation is strongly linked to the presence of amyloid plaques and tangles (Rogers et al., 1992; Shen et al., 2001). There is also an increase of complement receptors like C5aR in the brain (Ager et al., 2010). In AMD, the disease affects the central retina, where a build-up of debris is apparent under the neural retina (drusen) that contain complement proteins. In these conditions, the trigger for the underlying neuronal loss is different, and so is the spatial distribution of the tissue pathology, still the activation of the CS is a common feature.

In the brain, both glial cells and neurones are capable of producing CS proteins, especially after stimulation with cytokines (Gasque et al., 2000). Glial cells express complement inhibitors, 
while neurons express only low levels of complement control proteins, and therefore are at greater risk of homologous CS attack. Consequently, a sudden or progressive loss of neurons activates the complement system that is not sufficiently controlled and leads to further progression of the disease. Thus, inhibition of complement pathways is a focus for development of new therapeutic agents. These include modified native complement components e.g. sCR1, sDAF, and C1 inhibitor, complement inhibitory antibodies (antiC5 monoclonal ab), synthetic inhibitors (C5aR antagonists, factor $\mathrm{D}$ inhibitor) and other naturally occurring compounds (like heparin and cobra venom factor) (Bohlson et al., 2007). Most target the alternative pathway, since inhibition of the classical pathway may carry with it the risk of inducing lupus-like-conditions.

Here we review the recent research on these two important age-related diseases, and the focus is on the use of a complement inhibitor, the vaccinia virus complement control protein (VCP) to further elucidate the role of complement in CNS diseases and to evaluate whether complement inhibition has distinct advantages in being explored as a potential therapeutic target.

\section{Complement-The good and the bad}

The complement system is a well-defined host defense mechanism against invading microorganisms. It plays an important role in innate immunity of the host. Various components of complement are involved in inflammation. The activation of this critical system takes place through three pathways, the classical pathway of complement activation (CP), the alternative pathway of complement activation (AP), and lectin-mediated pathway (LMP). Out of these three, CP and AP are the major pathways of complement activation. Whenever a foreign molecule attacks the host, the complement system gets activated by either of these pathways. The antigen-antibody complex activates $\mathrm{C1q}$ component of the complement cascade and a series of actions are mediated leading to formation of $\mathrm{C} 3$ and $\mathrm{C} 5$ convertases, and subsequent production of anaphylatoxins. This pathway, may also be activated by the molecules other than antigen-antibody complexes (Veerhuis, 2011; Kotwal, 1997). AP, is activated by the surface of microorganisms and mucopolysaccharides. Or in the absence of microorganisms, it is activated by a control tick-over either by lowering the ability to control, or a higher rate of tick-over to overpower the controlling mechanisms which can lead to the uncontrolled activation that is found in the degenerative CNS (AD, AMD, Huntington's, Parkinson's, Multiple Sclerosis). The final consequence of complement activation by either of these pathways is the formation of membrane attack complex (MAC) and generation of anaphylatoxins. MAC is involved in the removal of foreign molecules or pathogens, and it also mediates cell lysis. The anaphylatoxins produce immediate immune responses through a series of reactions. They mediate their actions through chemotaxis and recruitment of leucocytes to the damaged area. The activation of leucocytes, macrophages, microglia and other brain cells mediated by the complement system, results in the expression of the adhesion molecules and results in their binding to the endothelial wall via the receptors on the endothelial cells, leading to the migration along the endothelial layer at the site of inflammation. The actions of the complement system under normal circumstances are controlled by complement regulatory proteins like C4b binding protein, CR1, DAF, CFH. The anaphylatoxins are also involved in vasodilation of vascular epithelium, by their direct actions or by mediating histamine release from mast cells. However, failure of these regulatory systems to control the activation of complement components results in damage (Daly and Kotwal, 1998).

\section{Complement in Alzheimer's disease (AD)}

Information combined from the fields of molecular biology, genetics, and histopathology suggest more complex picture of the pathological features associated with Alzheimer's disease (AD) (Selkoe, 2013). The development of suitable neuroprotective agents for the treatment of AD however, is still far from reality. The US FDA has approved 2 types of drugs: acetylcholinesterase inhibitors to prevent breakdown of acetylcholine, a neurotransmitter that is critical for learning and memory, for stages ranging from mild to moderate; and $\mathrm{N}$-methyl-D-aspartate (NMDA), a receptor antagonist for regulating glutamate activity for moderate to severe AD. In addition, non-steroidal anti-inflammatory drugs (NSAIDs) have been employed in the treatment of $\mathrm{AD}$ with clinical trials showing no special benefit. Herbs and dietary supplements like curcumin, melatonin, Vitamin E, an antioxidant and Ginkgo Biloba have been suggested to have benefits in general neuroprotection and slowing progression of memory impairment. However, there is no known cure for AD (Choi et al., 2014).

AD is associated with extracellular deposition of amyloid protein (Abeta1-42) and the formation of senile plaques in brain (Selkoe, 2001; Fan et al., 2007). As mentioned above, $\beta$-amyloid proteins have been demonstrated to induce complement activation (Rogers et al., 1992). Interestingly, the amyloid plaques have been shown to co-localise with microvessels, particularly in areas where haem-rich deposits were present. Consequently, it has been suggested, that Amyloid plaques are preferentially form in areas of micro-hemorrhages (Cullen et al., 2006). At such sites, the blood brain barrier most likely be compromised, allowing an invasion of inflammatory elements (macrophages, microglia) into the tissue (Crehan et al., 2012). In a model of age-related macular degeneration, Rutar et al. (2011a, 2011b) showed, that invading macrophages express complement and cause further cell damage, leading to progression of the disease.

The treatment of full-blown Alzheimer's disease has had limited and faltering success. It is now very clear that besides aging, genetic predisposition due to the presence of apo epsilon 4 (apoE4) alleles (one or two) and traumatic brain injury (TBI) to persons with apoE4 are major contributors to AD (Zlokovic, 2013; Liu et al., 2013; Mahley and Huang, 2012). Approximately, 60 million Americans ( 1 in 5 or $20 \%$ ) have at least one apoE4 allele and 6 million Americans or roughly $2 \%$ have 2 apoE4 alleles (the presence of two allele further increases the risk significantly). $35 \%$ of baby boomers in US, which accounts for about 21 million Americans, are currently predisposed to AD and have yet to progress to the terminal stages of $\mathrm{AD}$ and could receive health benefit in slowing that progression. Similar to AD, TBI also leads to activation of complement pathways and dementia. Wars and accidents have increased the number of TBI patients in whom the additional presence of apoE4 allele worsens the outcome. The connection between increased complement activation and apoE4 was first suggested by the groups of McGeer et al. (1997). In addition, the other complement-related genes identified as minor risk factors clusterin (CLU) and complement receptor 1 (CR1) contribute to the impaired regulation of the complement activation in these patients (Bagyinszky et al., 2014).

\section{Complement and macular degeneration}

Age-related macular degeneration (AMD) is a devastating degenerative disorder of the central retina that affects the elderly and is presently the leading cause of blindness among this population. AMD attacks the macula in the eye, leading to the loss of central vision that profoundly affects daily activities such as reading, driving and watching television (http://www.amd.org/). With a 
rising aging population, AMD is becoming a more profound medical concern for our society.

Inflammation and in particular the uncontrolled activation of the innate immune system have been linked to the progression of the disease. It is still unclear what is the initial cause of this activation, though it is believed that multiple factors play a role. Linkage studies showed that polymorphisms in genes of the complement system can modulate the susceptibility of individuals to the disease. To date, multiple genes have been linked to the disease, $\mathrm{C2}, \mathrm{CFB}, \mathrm{CFH}$ to mention a few (Gold et al., 2006; Edwards et al., 2005; Hageman et al., 2005; Gorin, 2005; Kuehn, 2005; Klein et al., 2005; Haines et al., 2005). Histological studies of diseased retinae are also indicative of complement system activation. The hallmark of the disease is the appearance of drusen (yellow spots) in and around the macula indicating the accumulation of debris under the retina. These drusen have been shown to contain dead photoreceptor cells, as well as inflammatory elements and complement proteins (Mullins et al., 2000).

Clinically, AMD is categorized as a dry or wet form. In the dry form (90\% of all cases), the accumulation of drusen and pigmentary changes, and the loss of photoreceptors characterize the slowly progressive disease, and in the end-stage it is described as geographic atrophy (GA). The wet form (10\% of cases) is named as such because of the presence of abnormal blood vessel growth behind the retina. The walls of these newly formed vessels are leaking fluid under the retina, causing macular edema and leading to the detachment of the central retina. Both forms of AMD are major causes of legal blindness in older people. AMD affects as many as 15 million Americans with more than 200,000 new cases each year that would be diagnosed. Presently, there is no known cure for the more common dry form, and while there are some treatment options for the wet form of the disease, they are limited to the reduction of blood vessel formation not addressing the underlying pathology. The use of antioxidants and vitamins has been recommended, but at best can only slow progression, if at all. Nonetheless, there are several drugs that are at early stage clinical trials for treatment of either dry or wet forms of AMD (Dong and Feng, 2007).

Given the strong link of the complement system and disease progression, one would assume that inhibition of complement pathways and inflammation may provide benefit to sufferers. As a result, complement pathways can be targeted for AMD treatments. In fact, one of the clinical trials that are currently undertaken is a C5b inhibitor (http://www.amd.org/) that suppresses the activities of complement pathways.

The CNS, that includes the retina, is an immune privileged site, and as such, leucocytes, macrophages are not normally present. Dendritic 'resting' microglia is responsible for the constant monitoring of changes in the retinal tissue, and these cells are the first to activate when changes, such as apoptosis occur. Activated microglia/macrophages clear the tissue of any debris. However, when cell death is more acute and in mass numbers, the signaling molecules released by the dying cells activate macroglia, (astrocytes in the brain, and the Muller cells in the retina). Studies in the retina showed, that Muller cells that are the main supporting cells responsible for the regulation of the retinal homeostasis, when stressed, express chemokines that recruit macrophages into the damaged region of the tissue (Rutar et al., 2010). In situ hybridization studies have shown, that invading macrophages express $\mathrm{C} 3$, and that they are also immunoreactive to C3 proteins (Rutar et al., 2011a, 2011b). The presence of complement in the damaged retina, and more importantly in the penumbra surrounding the most severe injury site, suggests a chronic inflammatory state that results in a self-perpetuating loss of neighboring photoreceptors, thus leading to disease progression.

\section{Complement regulation-The challenge of finding the right therapy}

Several drugs are currently in the pipeline that targets the neurodegenerative effects of beta-amyloid accumulation. However, most of the drugs in trial are either ineffective or have other significant problems. Large pharmaceutical companies have been spending hundreds of millions dollars on clinical trials and on acquiring the R\&D pipelines of small biotech companies with promising products. In the long term, physicians and sectors of the pharmaceutical and biotech industry are in search of newer and more effective options to prevent or slow AD. It is well established that amyloid proteins and plaques are associated with activation of several pro-inflammatory mediators, especially complement components (Veerhuis, 2011). These pro-inflammatory mediators cause damage on their own, and also work in synergy with each other, ultimately leading to neurodegeneration. Over time, the damaging role of the complement system overpowers its beneficial effects as the disease progresses. One might wonder if complement pathways, distinct from other pro-inflammatory pathways can be targeted for blocking of disease progression of AD.

A major secretory pox viral protein termed vaccinia virus complement control protein (VCP) was found to inhibit the complement system (Kotwal and Moss, 1988). VCP regulates both the classical pathway of complement activation (CP), and the alternative pathway of complement activation (AP). VCP binds to the fourth component of complement ( $\mathrm{C} 4 \mathrm{~b})$ at low dose and to the third component ( $\mathrm{C} 3 \mathrm{~b}$ ) at higher doses. It is known to inhibit $\mathrm{C} 3$ convertases and subsequent production of anaphylotoxins $\mathrm{C} 3 \mathrm{a}, \mathrm{C} 4 \mathrm{a}$, and $\mathrm{C} 5 \mathrm{a}$. VCP shows the therapeutic potential that will fit a market need for a product that can specifically modulate complement-mediated neuroinflammation due to undesirable and excessive complement activation, thereby neurodegeneration in AD (Daly and Kotwal, 1998). Compstatin, a complement inhibitor developed during the same time was also well on its way to clinical application (Ricklin and Lambris, 2008). Another treatment possibility is a C5aR antagonist tested in murine models of Alzheimer's disease (Fonseca et al., 2009).

\section{Evidence from the complement regulation by VCP in the brain, prior to the onset of $A D$}

There could be considerable benefit from early intervention using VCP (Kotwal, 2011). Preclinical studies in AD models with VCP(Pillay et al., 2008; Kulkarni et al., 2008) demonstrated valuable promise. However, the obstacle to the realization of the unparalleled potential of VCP is the blood-brain barrier. This obstacle can be only overcome if an effective delivery method of VCP can be developed. Although in the case of Traumatic brain injury the blood brain is compromised and VCP could gain entry when injected intravenously but in the case of $\mathrm{AD}$, this may not happen reliably.

The first novel candidate, VCP has had extensive preclinical studies published in peer reviewed scientific publications, to be proposed to be one that would block the progression not by eliciting a protective immune response (such as in the case of attempts at a vaccine) but by blocking the complement mediated neuroinflammation, that would result from the formation of Abeta plaques and the tau tangles. VCP has shown promise in rodent models of $\mathrm{AD}$ and TBI discussed later. Earlier data indicates that VCP binds to the third component of the complement system, making it a very potent and effective molecule for the control of complement activation. VCP has several unique advantages over potential competitive products: VCP is one of the smallest natural complement control proteins in size, is the only complement inhibitor directly targeting the components of the complement system, and to have 


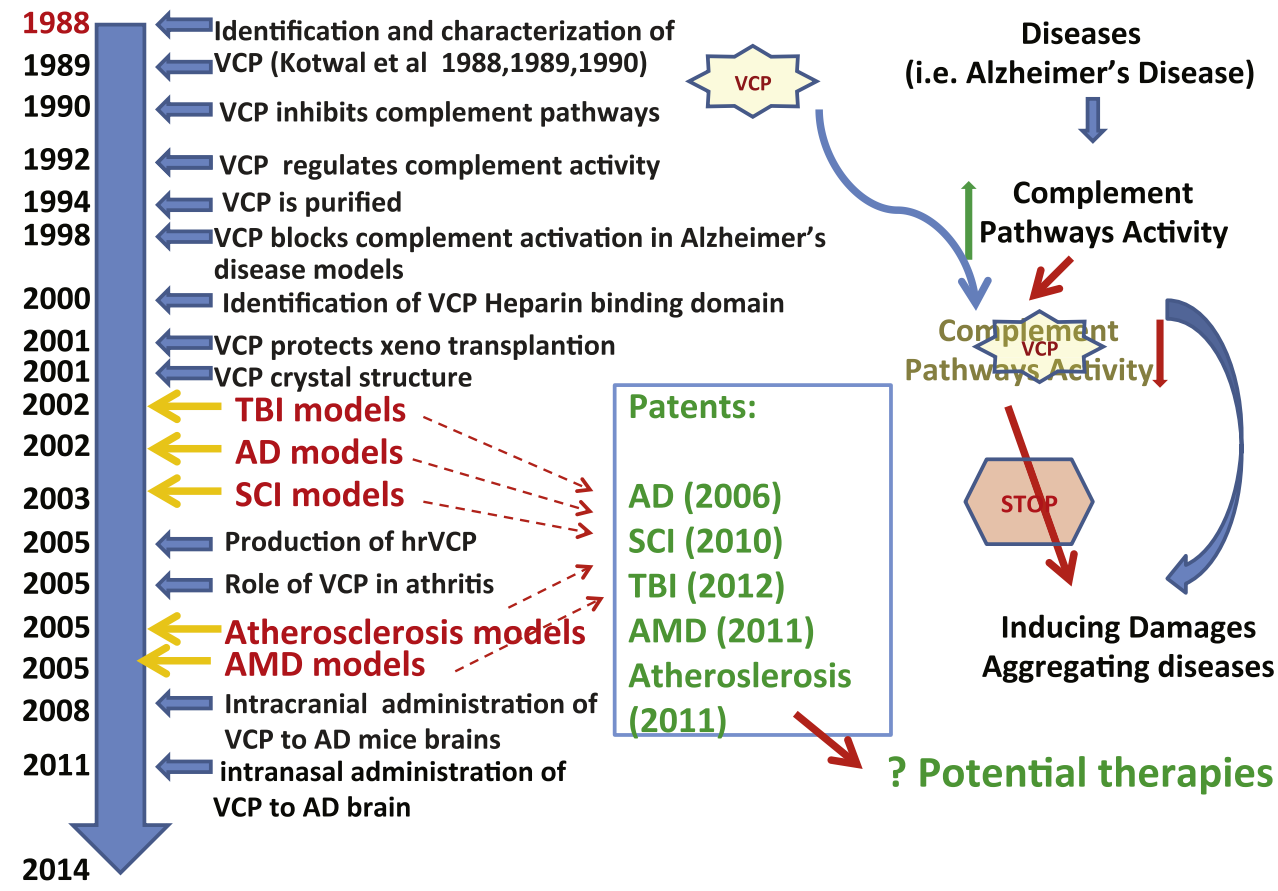

Fig. 1. Timeline of VCP research.

been evaluated in therapeutic models of spinal cord injury (SCI), TBI and AD with promising results (Hicks et al., 2002; Reynolds et al., 2004; Pillay et al., 2007; Kulkarni et al., 2008, 2011). VCP is a robust protein that is heat, $\mathrm{pH}$, and freeze-thaw stable; inactivated only by boiling (Smith et al., 2002). VCP can be purified (Kotwal, 1994; Murthy et al., 2001), stored and transported very easily at room temperature. It can be easily converted into dosage forms. VCP has high tissue retention due to its heparin binding ability (Smith et al., 2000). In preclinical studies, VCP has been safely administered to rodents and baboons with no observed toxicity (Anderson et al., 2002; Kahn et al., 2003). VCP, purified from natural infection with vaccinia virus infected cells and from the medium of recombinant yeast Pichia pastoris, has been extensively characterized over the past twenty years. VCP inhibits both the classical and alternate complement pathways (Kotwal and Moss, 1988; Kotwal et al., 1990). Previous studies with VCP suggest, a beneficial role of VCP in CNS disorders, especially in AD, SCI and TBI (Pillay et al., 2004, 2008; Reynolds et al., 2004; Kulkarni et al., 2008). In more recent reports in rodent behavior studies (Kulkarni et al., 2008, 2011; Pillay et al., 2008). VCP has been demonstrated to have improved memory outcomes and diminished anxiety at later stages of transgenic AD mice when intranasal injections were made at early stages and shown to be distributed throughout the CNS (Kulkarni et al., 2012).

\section{Evidence from the retina degeneration models demonstrating the protective role of VCP and compstatin}

The role of complement regulation by VCP in degenerative diseases of the eye has been recently investigated in 2 models of retinal degeneration. A model of dry AMD is the light-induced retinal degeneration in albino rats. In this model, bright continuous light causes photoreceptor specific damage and death. The photoreceptor cell death is closely followed by the production of cytokines by the activated Muller cells and the recruitment of macrophages to the site of damage. The invading macrophages deposit complement proteins to the site and the surrounding areas, causing the progressive loss of neighbouring photoreceptors, even after the removal of the initial stress (bright white light) (Rutar et al., 2010, 2011a,b).
In this study the first investigations have started to establish the uptake of VCP into the retina following intravitreal injections and has been confirmed, that VCP reaches all layers of the retina shortly after the injection. However, because of its fast removal by the rapid blood flow in the tissue and choroid, the use of slow release formulas are being tested for extended delivery of VCP to the retina (Fernando and Valter, unpublished). The wet form of AMD is studied in a well-established laser-induced choroidal neovascularisation $(\mathrm{CNV})$ rodent model. In these studies, krypton red laser photocoagulation is used to generate multiple tears in the Blood retina barrier, initiating the production of inflammatory cytokines and chemokines as well as the activation of the complement system. VCP injected in to the eye, 1-4 days after the laser shots showed to reduce the presence of vascular changes (Francois et al., 2006).

Novel compstatin family of peptides, inhibitors of complement activation have also has also been investigated in a cell culture model of human retinal pigmented epithelial cells where it was demonstrated to inhibit complement activation by drusen-like deposits (Gorham et al., 2013) and in cynomolgus monkeys with early-onset macular degeneration (2010).

\section{Summary/conclusion}

VCP promises precisely the therapeutic potential that would fill the need for a product that could modulate neuroinflammation due to undesirable and excessive complement activation. VCP is structurally similar to human complement regulatory proteins, and our studies in a range of animal models have shown that it does not elicit any significant immune response that could reduce its effectiveness. Finally, it needs to be emphasized that after 25 years since its discovery (Fig. 1), VCP remains the only candidate that has been reported to have a full range of complement regulatory activities proposed as a prophylactic molecule to accomplish neuroprotection in AD and macular degeneration. In AMD, all other small compounds, small peptides, antibodies to complement components [POT-4 (Compstatin), eculizumab, tandospirone, anecortave acetate, the antioxidant OT-551, sirolimus] have not been shown to be effective in human trials (Leung and Landa, 2013). 
Recently, there has been intense activity to overcome the shortcomings of compstatin. Several new analogues more active than the original compstatin analogue, POT-4, used in clinical trial have been developed (Knerr et al., 2011; Tamamis et al., 2012). Besides proteins and circular polypeptides, nucleic acids (miRNA) are also being considered as therapeutic agents in regulating complement receptors (Lukiw and Alexandrov, 2012). In conclusion, modulation of complement could be a therapeutic target by a number of possible candidates for achieving neuroprotection from neuroinflammation.

\section{Acknowledgement}

The major funding for the VCP research reviewed in the manuscript was from the Wellcome Trust of England and the Kentucky Spinal Cord and Head Injury Research Trust, Kentucky, USA. Funding from National Health \& Medical Research Council of Australia (NHMRC) and the Australian Research Council (ARC) was awarded to KV for studying role of complement in retinal diseases. The authors would like to dedicate this review in memory of our distinguished colleague and collaborator Dr. Gudmundur Johann Arason from Iceland who passed away on April 09, 2014.

\section{References}

Ager, R.R., Fonseca, M.I., Chu, S.H., Sanderson, S.D., Taylor, S.M., Woodruff, T.M., Tenner, A.J., 2010. Microglial C5aR (CD88) expression correlates with amyloidbeta deposition in murine models of Alzheimer's disease. J. Neurochem. 113 (2), 389-401.

Alexander, J.J., Anderson, A.J., Barnum, S.R., Stevens, B., Tenner, A.J., 2008. The complement cascade: Yin-Yang in neuroinflammation-neuro-protection and -degeneration. J. Neurochem. 107 (5), 1169-1187.

Anderson, J.B., Smith, S.A., Kotwal, G.J., 2002. Vaccinia virus complement control protein inhibits hyperacute xenorejection. Transplant. Proc. 34 (4), 1083-1085.

Bagyinszky, E., Youn, Y.C., Kin, S.Y., 2014. The genetics of Alzheimer's disease. Clin. Interv. Aging 9, 535-551.

Bohlson, S.S., Fraser, D.A., Tenner, A.J., 2007. Complement proteins C1q and MBL are pattern recognition molecules that signal immediate and long-term protective immune functions. Mol. Immunol. 44, 33-43.

Choi, S.S., lee, S.-R., Lee, H.-J., 2014. Alzheimer's disease and stem cell therapy. Exp. Neurobiol. 23 (1), 45-52

Crehan, H., Hardy, J., Pocock, J., 2012. Microglia Alzheimer's disease, and complement. Int. J. Alzheimers Dis. 2012, 983640, http://dx.doi.org/10.1155/2012/983640, Epub 2012 Aug21. PubMed PMID: 22957298; PubMed Central PMCID: PMC3432348.

Cullen, K.M., Kocsi, Z., Stone, J., 2006. Microvascular pathology in the aging human brain: evidence that senile plaques are sites of microhaemorrhages. Neurobiol. Aging 27 (12), 1786-1796.

Daly 4th, J., Kotwal, G.J., 1998. Pro-inflammatory complement activation by the A beta peptide of Alzheimer's disease is biologically significant and can be blocked by vaccinia virus complement control protein. Neurobiol. Aging 19 (6 (Nov-Dec)), 619-627.

Dong, Y., Feng, S.S., 2007. Poly(D L-lactide-co-glycolide) (PLGA) nanoparticles prepared by high pressure homogenization for paclitaxel chemotherapy. Int. J. Pharm. 342 (1-2), 208-214.

Edwards, A.O., Ritter 3rd, R., Abel, K.J., Manning, A., Panhuysen, C., Farrer, L.A., 2005. Complement factor $\mathrm{H}$ polymorphism and age-related macular degeneration. Science 308 (5720 (Apr)), 421-424

Fan, R., DeFilippis, K., Van Nostrand, W.E., 2007. Induction of complement proteins in a mouse model for cerebral microvascular A beta deposition. J. Neuroinflammation 4, 22.

Fonseca, M.I., Ager, R.R., Chu, S.H., Yazan, O., Sanderson, S.D., LaFerla, F.M., Taylor, S.M., Woodruff, T.M., Tenner, A.J., 2009. Treatment with a C5aR antagonist decreases pathology and enhances behavioral performance in murine models of Alzheimer's disease. J. Immunol. 183 (2), 1375-1383.

Francois, C., Deschatelets, P., Olson, P., 2006. Viral complement control proteins for eye disorders. In: Unites States Patent Application Publication Pub. No. US 2006/0142191 A1.

Gasque, P., Dean, Y.D., et al., 2000. Complement components of the innate immune system in health and disease in the CNS. Immunopharmacology 49, 171-186.

Gorin, M.B., 2005. A new vision for age-related macular degeneration. Eur. J. Hum. Genet. 13 (7 (Jul)), 793-794.

Gorham Jr., R.D., Forest, D.L., Tamamis, P., López de Victoria, A., Kraszni, M., Kieslich, C.A., Banna, C.D., Bellows-Peterson, M.L., Larive, C.K., Floudas, C.A., Archontis, G., Johnson, L.V., Morikis, D., 2013. Novel compstatin family peptides inhibit complement activation by drusen-like deposits in human retinal pigmented epithelial cell cultures. Exp. Eye Res. 2013 (116), 96-108.
Gold, B., Merriam, J.E., Zernant, J., Hancox, L.S., Taiber, A.J., Gehrs, K., Cramer, K. Neel, J., Bergeron, J., Barile, G.R., Smith, R.T., AMD Genetics Clinical Study Group, Hageman, G.S., Dean, M., Allikmets, R., 2006. Variation in factor B (BF) and complement component 2 (C2) genes is associated with age-related macular degeneration. Nat. Genet. 38 (4 (Apr)), 458-462.

Hageman, G.S., Anderson, D.H., Johnson, L.V., Hancox, L.S., Taiber, A.J., Hardisty, L.I. Hageman, J.L., Stockman, H.A., Borchardt, J.D., Gehrs, K.M., Smith, R.J., Silvestri, G., Russell, S.R., Klaver, C.C., Barbazetto, I., Chang, S., Yannuzzi, L.A., Barile, G.R Merriam, J.C., Smith, R.T., Olsh, A.K., Bergeron, J., Zernant, J., Merriam, J.E., Gold, B., Dean, M., Allikmets, R., 2005. A common haplotype in the complement regulatory gene factor $\mathrm{H}$ (HF1/CFH) predisposes individuals to age-related macular degeneration. Proc. Nat. Acad. Sci. U.S.A. 102 (20 (May)), 7227-7232.

Haines, J.L., Hauser, M.A., Schmidt, S., Scott, W.K., Olson, L.M., Gallins, P., Spencer, K.L. Kwan, S.Y., Noureddine, M., Gilbert, J.R., Schnetz-Boutaud, N., Agarwal, A., Postel, E.A., Pericak-Vance, M.A., 2005. Complement factor $\mathrm{H}$ variant increases the risk of age-related macular degeneration. Science 308 (5720 (Apr)), 419-421.

Hicks, R.R., Keeling, K.L., Yang, M.Y., Smith, S.A., Simons, A.M., Kotwal, G.J., 2002 Vaccinia virus complement control protein enhances functional recovery after traumatic brain injury. J. Neurotrauma 19 (6), 705-714.

Kahn, D., Smith, S.A., Kotwal, G.J., 2003. "Dose-dependent inhibition of complement in baboons by vaccinia virus complement control protein: implications in xenotransplantation. Transplant. Proc. 35 (4), 1606-1608.

Klein, R.J., Zeiss, C., Chew, E.Y., Tsai, J.Y., Sackler, R.S., Haynes, C., Henning, A.K., SanGiovanni, J.P., Mane, S.M., Mayne, S.T., Bracken, M.B., Ferris, F.L., Ott, J., Barnstable, C., Hoh, J., 2005. Complement factor $\mathrm{H}$ polymorphism in age-related macular degeneration. Science 15 (308 (Apr)), 385-389, 5720.

Knerr, P.J., Tzekou, A., Ricklin, D., Qu, H., Chen, H., van der Donk, W.A., Lambris, J.D., 2011. Synthesis and activity of thioether-containing analogues of the complement inhibitor compstatin. ACS Chem. Biol. 6 (7), 753-760.

Kotwal, G.J., 1994. Purification of virokines using ultrafiltration. Am. Biotechnol. Lab. $12(10), 76-77$.

Kotwal, G.J., Isaacs, S.N., McKenzie, R., Frank, M.M., Moss, B., 1990. Inhibition of the complement cascade by the major secretory protein of vaccinia virus. Science 250 (4982), 827-830.

Kotwal, G.J., Moss, B., 1988. Vaccinia virus encodes a secretory polypeptide structurally related to complement control proteins. Nature 335 (6186), 176-178.

Kotwal, G.J., 1997. Microorganisms and their interaction with the immune system. J. Leukoc. Biol. 62 (4 (Oct)), 415-429.

Kotwal, G.J., 2011. Complement-initiated neuroinflammation and its role in early stage Alzheimer's disease. Commentary. Curr. Alzheimer Res. 8 (1 (Feb)), 32-33, PubMed PMID: 21294703.

Kuehn, B.M., 2005. Gene discovery provides clues to cause of age-related macular degeneration. JAMA 293 (15), 1841-1845.

Kulkarni, A.P., Govender, D.A., Kotwal, G.J., Kellaway, L.A., 2011. Modulation of anxiety behavior by intranasally administered vaccinia virus complement contro protein and curcumin in a mouse model of Alzheimer's disease. Curr. Alzheimer Res. 8 (1), 95-113.

Kulkarni, A.P., Pillay, N.S., Kellaway, L.A., Kotwal, G.J., 2008. Intracranial administration of vaccinia virus complement control protein in Mo/Hu APPswe PS1dE9 transgenic mice at an early age shows enhanced performance at a later age using a cheese board maze test. Biogerontology 9 (6), 405-420.

Kulkarni, A.P., Govender, D., Kellaway, L.A., Kotwal, G.J., 2012. Central nervous system distribution of the poxviral proteins after intranasal administration of proteins and titering of vaccinia virus in the brain after intracranial administration. Methods Mol. Biol. 890, 305-326.

Leung, E., Landa, G., 2013. Update on current and future novel therapies for dry agerelated macular degeneration. Expert Rev. Clin. Pharmacol. 6 (5 (Sep)), 565-579.

Liu, C.C., Kanekiyo, T., Xu, H., Bu, G., 2013. Apolipoprotein E and Alzheimer disease: risk, mechanisms and therapy. Nat. Rev. Neurol. 9 (2 (Feb)), 106-118, http://dx.doi.org/10.1038/nrneurol.2012.263, Epub 2013 Jan 8. Erratum in: Nat Rev Neurol. 2013.doi: 10.1038/nmeurol.2013.32. Liu, Chia-Chan [corrected to Liu, Chia-Chin].

Lukiw, W.J., Alexandrov, P.N., 2012. Regulation of complement factor H (CFH) by multiple miRNAs in Alzheimer's disease (AD) brain. Mol. Neurobiol. 46 (1), 11-19, Review.

Mahley, R.W., Huang, Y., 2012. Apolipoprotein e sets the stage: response to injury triggers neuropathology. Neuron 76 (5 (Dec)), 871-885, http://dx.doi.org/ 10.1016/j.neuron.2012.11.020, Review. PubMed PMID:23217737.

McGeer, P.L., Walker, D., Pitas, R.E., Mahley, R.W., McGeer, E.G., 1997. Apolipoprotein (Apo E4 but not Apo E or Apo E2 potentiates Beta amyloid protein activation of complement in vitro. Brain Res. 749, 135-138.

Mullins, R.F., Russell, S.R., Anderson, D.H., Hageman, G.S., 2000. Drusen associated with aging and age-related macular degeneration contain proteins common to extracellular deposits associated with atherosclerosis, elastosis, amyloidosis, and dense deposit disease. FASEB J. 14 (7 (May)), 835-846.

Murthy, K.H., Smith, S.A., Ganesh, V.K., Judge, K.W., Mullin, N., Barlow, P.N., Ogata C.M., Kotwal, G.J., 2001. Crystal structure of a complement control protein that regulates both pathways of complement activation and binds heparan sulfate proteoglycans. Cell 104 (2), 301-311.

Pillay, N.S., Kellaway, L.A., Kotwal, G.J., 2004. Molecular mechanisms, emerging etiological insights and models to test potential therapeutic interventions in Alzheimer's disease. Curr. Alzheimer Res. 1 (4), 295-306.

Pillay, N.S., Kellaway, L.A., Kotwal, G.J., 2007. Vaccinia virus complement control protein significantly improves sensorimotor function recovery after severe head trauma. Brain Res. 1153, 158-165. 
Pillay, N.S., Kellaway, L.A., Kotwal, G.J., 2008. Early detection of memory deficits and memory improvement with vaccinia virus complement control protein in an Alzheimer's disease model. Behav. Brain Res. 192 (2), 173-177.

Ricklin, D., Lambris, J.D., 2008. Compstatin: a complement inhibitor on its way to clinical application. Adv. Exp. Med. Biol. 632, 273-292, Review.

Reynolds, D.N., Smith, S.A., Zhang, Y.P., Mengsheng, Q., Lahiri, D.K., Morassutti, D.J., Shields, C.B., Kotwal, G.J., 2004. Vaccinia virus complement control protein reduces inflammation and improves spinal cord integrity following spinal cord injury. Ann. N.Y. Acad. Sci. 1035, 165-178.

Rogers, J., Cooper, N.R., Webster, S., Schultz, J., McGeer, P.L., Styren, S.D., Civin, W.H., Brachova, L., Bradt, B., Ward, P., et al., 1992. Complement activation by betaamyloid in Alzheimer disease. Proc. Nat. Acad. Sci. U.S.A. 89 (21), 10016-10020, PubMed PMID: 1438191 PubMed Central PMCID: PMC50268.

Rutar, M., Natoli, R., Kozulin, P., Valter, K., Gatenby, P., Provis, J.M., 2011a. Analysis of complement expression in light-induced retinal degeneration: synthesis and deposition of C3 by microglia/macrophages is associated with focal photoreceptor degeneration. Invest. Ophthalmol. Vis. Sci. 52 (8 (Jul)), 5347-5358, http://dx.doi.org/10.1167/iovs.10-7119, PubMed PMID: 21571681.

Rutar, M., Natoli, R., Valter, K., Provis, J.M., 2011b. Early focal expression of the chemokine $\mathrm{Ccl} 2$ by Müller cells during exposure to damage-inducing bright continuous light. Invest. Ophthalmol. Vis. Sci. 52 (5), 2379-2388.

Rutar, M., Provis, J.M., Valter, K., 2010. Brief exposure to damaging light causes focal recruitment of macrophages, and long-term destabilization of photoreceptors in the albino rat retina. Curr. Eye Res. 35 (7), 631-643.

Selkoe, D.J., 2001. Clearing the brain's amyloid cobwebs. Neuron 32 (2), 177-180.

Selkoe, D.J., 2013. SnapShot: pathobiology of Alzheimer's disease. Cell 154 (2 (Jul)), 468-468.

Shen, Y., Lue, L., Yang, L., Roher, A., 2001. Complement activation by neurofibrillary tangles in Alzheimer's disease. Neurosci. Lett. 305 (3), 165-168.
Singhrao, S.K., Neal, J.W., Morgan, B.P., Gasque, P., 1999. Increased complement biosynthesis by microglia and complement activation on neurons in Huntington's disease. Exp. Neurol. 159 (2), 362-376.

Smith, S.A., Krishnasamy, G., Murthy, K.H., Cooper, A., Bromek, K., Barlow, P.N., Kotwal, G.J., 2002. Vaccinia virus complement control protein is monomeric, and retains structural and functional integrity after exposure to adverse conditions. Biochim. Biophys. Acta 1598 (1-2), 55-64.

Smith, S.A., Mullin, N.P., Parkinson, J., Shchelkunov, S.N., Totmenin, A.V., Loparev, V.N., Srisatjaluk, R., Reynolds, D.N., Keeling, K.L., Justus, D.E., Kotwal, G.J., 2000. Conserved surface-exposed K/R-X-K/R motifs and net positive charge on poxvirus complement control proteins serve as putative heparin binding sites and contribute to inhibition of molecular interactions with human endothelial cells: a novel mechanism for evasion of host defense. J. Virol. 74 (12), 5659-5666.

Stephan, A.H., Madison, D.V., Mateos, J.M., Fraser, D.A., Lovelett, E.A., Coutellier, L. Kim, L., Tsai, H.H., Huang, E.J., Rowitch, D.H., Berns, D.S., Tenner, A.J., Shamloo, M., Barres, B.A., 2013. A dramatic increase of C1q protein in the CNS during norma aging. J. Neurosci. 33 (33), 13460-13474.

Tamamis, P., López de Victoria, A., Gorham Jr., R.D., Bellows-Peterson, M.L., Pierou, P., Floudas, C.A., Morikis, D., Archontis, G., 2012. Molecular dynamics in drug design: new generations of compstatin analogs. Chem. Biol. Drug Des. 79 (5 (May)), 703-718.

Veerhuis, R., 2011. Histological and direct evidence for the role of complement in the neuroinflammation of AD. Curr. Alzheimer Res. 8 (1), 34-58.

Zhou, J., Fonseca, M.I., Pisalyaput, K., Tenner, A.J., 2008. Complement C3 and C4 expression in C1q sufficient and deficient mouse models of Alzheimer's disease. J. Neurochem. 106 (5), 2080-2092.

Zlokovic, B.V., 2013. Cerebrovascular effects of apolipoprotein E: implications for Alzheimer disease. JAMA Neurol. 70 (4 (Apr)), 440-444, Review. 